\title{
Editorial: Smarter Farming: New Approaches for Improved Monitoring, Measurement and Management of Agricultural Production and Farming Systems
}

\author{
Matt J. Bell ${ }^{1 *}$ and Alexis Comber ${ }^{2}$ \\ ${ }^{1}$ School of Biosciences, The University of Nottingham, Sutton Bonington, United Kingdom, ${ }^{2}$ School of Geography, Leeds \\ Institute for Data Analytics, University of Leeds, Leeds, United Kingdom
}

Keywords: farming, food production, precision management, information technologies, big data

\section{Editorial on the Research Topic}

Smarter Farming: New Approaches for Improved Monitoring, Measurement and Management of Agricultural Production and Farming Systems

There is considerable interest and investment in the information that can be derived from new technologies and data to support enhanced monitoring, measurement and management of farming systems for more sustainable food production. At the farm level, information on aspects associated with animals, plants, soil, water, and the farm environment must be in a useful format to enhance management. For example, identifying changes to improve production efficiency (increased outputs and reduced inputs) and profitability of food products is of great interest to farmers, and also has the ability to confer efficiency savings by potentially reducing the environmental impact of production. Nutrient losses from agricultural land negatively impact water and air quality. The papers of Comber et al., McNunn et al. and Preza Fontes et al. discussed tools for enhanced land management to assist with managing nutrient losses from agricultural land. Agriculture is a significant source of water and air pollution. In addition to the environmental benefits of improved nutrient management, the efficacy of any agricultural application is severely reduced if poorly timed and it washes from the crop or the field into watercourses. This reduced efficacy leads to risks of reduced output (i.e., crop yields) and increased input costs (i.e., sprays and fertilizer). McNunn et al. suggest that managing cropping systems for the economic optimum will likely lead to improved environmental outcomes when modeling nitrogen losses from cropping systems. Preza Fontes et al. showed that remote sensing vegetation indices were correlated with nitrous oxide emissions, indicating that new technologies (e.g., unmanned aerial vehicle platform) could represent an integrative tool for linking sustainability outcomes with improved agronomic efficiencies; with lower vegetation index values associated with poor crop performance and higher nitrous oxide emissions. The authors also discovered that the use of an unmanned aerial vehicle to evaluate water quality was limited due to the timing of nutrient losses, which happened prior to early-season crop growth and image collection. Comber et al. provided a modeling framework that can be used to identify hotspots within fields and watercourses, with the aim of supporting informed on-the-ground catchment management by environmental agencies and water companies. Tools that can enhance productivity and reduce environmental impact are of great importance to policy makers and wider society. 
Information and data from real-time and automated systems can also help early detection and improve awareness of poor performance, thereby allowing farmers to make timely and informed interventions and changes in practice to enhance the efficiency and sustainability of production. As financial pressures on farmers increase, each farm worker will be expected to allocate their time effectively and toward the tasks that need their attention most. However, the potential usefulness of new technologies, their integration into decision support tools and how such systems may complement other or existing information for food production is still being understood. Bell and Tzimiropoulos discussed how production efficiencies associated with resource utilization and monitoring of animal traits have reduced the environmental impact of cattle systems. Papers of Bell et al. and Miller et al. demonstrate promising imaging technologies to estimate dairy cow body condition (particularly identify low or high body fat) and beef cattle liveweight and carcass characteristics, respectively. Camera monitoring systems have the benefit of providing objective information and not relying on human intervention, transponder attachments, or invasive equipment (e.g., boluses, collars). This can be particularly important for the accuracy of body condition scoring, with digital imaging providing more accuracy compared to manual and subjective scoring methods in cows with a low body condition (Bell et al.). Images can provide lots of opportunities for information (e.g., animal and plant health) at a relatively low cost. Combining tools and sources of digital information may ultimately provide more complete and enhanced monitoring systems for farm use, such as developments in pasture cover (using mobile measures of plant biomass) and pasture nutrient concentrations (using mobile near-infrared spectroscopy) measured in real-time as shown in the paper of Bell et al. Changes in pasture nutrients are typically not monitored but doing so may help land managers improve how effectively they manage forage, which is an important source of nutrients for ruminant livestock and biodiversity in our rural landscape.

While it is acknowledged that there are increasing opportunities to use smart farming technologies and data-driven decision making for improved management of food production systems, the papers of Ingram and Maye, and Eastwood and Renwick, highlighted that we need to better understand the wider issues affecting a farmer's uptake of such smart farming technology and information. Ingram and Maye discussed the "fourth agricultural revolution" of digital agriculture and the implications of digitalization for agricultural knowledge. Digital applications and platforms have the potential to dramatically change the way knowledge is processed, communicated, accessed and utilized as farming processes become increasingly data-driven and data-enabled. The authors proposed that this raises critical questions about how digital agriculture will require new capabilities, support decision-making and interact with, and potentially disrupt, established modes of knowledge processing between people and organizations in these multi-actor knowledge networks i.e., Agricultural Knowledge Innovation Systems. An understanding of the implications of new digital information are important for effective implementation, from support for farmers to data analytics and the linkages between actors. Eastwood and Renwick investigated the impact of innovation uncertainty on adoption of automatic milking systems (AMS) and showed that minimizing the uncertainty around the innovation can influence its success. Eastwood and Renwick highlighted the potential impact of negative experiences associated with new technologies from farmers who struggle with the adaptation process, as such occurrences may act to stall the uptake of smart farming technologies. The authors propose that if public policy organizations are to realize the desired impacts of smart farming technology, there needs to be greater focus on understanding where (and which) technologies can have an actual impact on farm, and greater public and private $\mathrm{R} \& \mathrm{D}$ collaboration is required to foster knowledge development and exchange.

\section{AUTHOR CONTRIBUTIONS}

MB wrote the editorial. AC assisted with editing. Both authors contributed to the article and approved the submitted version.

Conflict of Interest: The authors declare that the research was conducted in the absence of any commercial or financial relationships that could be construed as a potential conflict of interest.

Copyright (c) 2020 Bell and Comber. This is an open-access article distributed under the terms of the Creative Commons Attribution License (CC BY). The use, distribution or reproduction in other forums is permitted, provided the original author(s) and the copyright owner(s) are credited and that the original publication in this journal is cited, in accordance with accepted academic practice. No use, distribution or reproduction is permitted which does not comply with these terms. 